\title{
Accurate theoretical fits to laser-excited photoemission spectra in the normal phase of high-temperature superconductors
}

\author{
PHILIP A. CASEY ${ }^{1}$, J. D. KORALEK ${ }^{2,3}$, N. C. PLUMB² , D. S. DESSAU ${ }^{2,3}$ AND PHILIP W. ANDERSON ${ }^{1 *}$ \\ ${ }^{1}$ Department of Physics, Princeton University, Princeton, New Jersey 08544, USA \\ ${ }^{2}$ Department of Physics, University of Colorado, Boulder, Colorado 80309, USA \\ ${ }^{3}$ JILA, University of Colorado and NIST, Boulder, Colorado 80309, USA \\ *e-mail: pwa@princeton.edu
}

It has long been believed that the 'normal' (nonsuperconducting) state of the high-transition-temperature superconductors is anything but normal ${ }^{1-3}$. In particular, this state, which exists above the superconducting transition temperature $T_{c}$, has very unusual transport properties ${ }^{4,5}$ and electron spectral functions ${ }^{6,7}$, presenting a more difficult, complex and important problem than the superconductivity itself. The origin of this difficulty and complexity resides in the strong electronic correlations, or the many-body Coulomb interactions between electrons, which cannot be properly treated within the standard theories of the electronic structure of solids. A new treatment of these interactions, on the basis of a Gutzwiller projection-which gives zero quasiparticle weight at the Fermi surface and removes the possibility for double electron occupancy on any one sitehas recently been proposed ${ }^{8}$, but fits to available data were unsatisfactory. Here, we compare the electron spectral functions computed within this theoretical treatment with bulk-sensitive measurements made by low-energy photons, using laserexcited angle-resolved photoemission spectroscopy of the superconductor $\mathrm{Bi}_{2} \mathrm{Sr}_{2} \mathrm{CaCu}_{2} \mathrm{O}_{8+\delta}$ (refs 9,10). The theory captures the asymmetrical shape of the experimental curves with good accuracy and in principle has only one free parameter. Moreover, no background subtraction is necessary.

The most direct way to measure the electronic excitation spectrum of a solid is angle-resolved photoemission spectroscopy (ARPES), which gives the energy- and momentum-dependent spectral function ${ }^{11}$. Historically, results of these measurements on cuprate superconductors have given broad spectral features as well as large backgrounds $s^{6,7}$. These features were not well understood, but generically have been described as representative of short-lived electronic states or totally incoherent electronic states such as would be the case in a system with spin-charge separation. Recently however, much sharper 'quasiparticle-like' electronic excitations have been observed using the laser-excited ARPES technology developed in Colorado, ${ }^{9,10}$. The low photon energy in these experiments $(6 \mathrm{eV}$ as compared with the typical $20-50 \mathrm{eV}$ ) greatly increases both the momentum and energy resolution, reduces the final-state broadening effects, decreases the background and increases the bulk sensitivity, each of which overcomes concerns of the previous experiments ${ }^{9,10}$. Thus, it is believed that the new

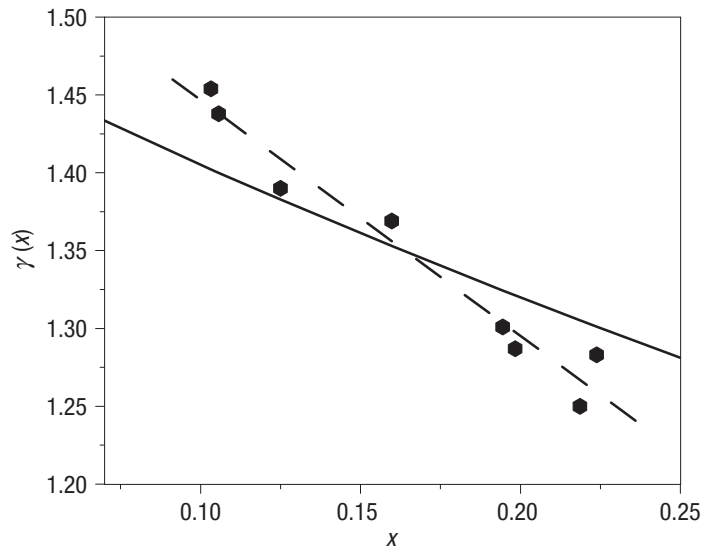

Figure 1 Infrared spectrum exponents for $\mathrm{Bi}_{2} \mathrm{Sr}_{2} \mathrm{CaCu}_{2} \mathrm{O}_{8+\delta}$. Data points from ref. 13 with linear best fit of ref. 13 (dashed line) and predicted value from ref. 8 (solid line). The predicted exponent stems from $\sigma(\omega)=(i \omega)^{-2+\gamma}$ with $\gamma=1+2 p$, and $p$ is given in the text.

laser-excited ARPES spectra represent the most accurate cuprate spectral line shapes so far. A theoretical understanding of these spectra is clearly required.

One of the most difficult aspects of developing a theory of the electronic structure of the cuprate superconductors is dealing with the coulombic interactions between the doped holes (absence of electrons, which are the carriers of electric current). In particular, a double occupancy of holes on a single lattice site is energetically unfavourable compared with single or zero occupancy, a fact that breaks the electron-hole symmetry about the half-filled case. In 1963, Gutzwiller presented his projection operator to deal with the Coulomb interactions, by which the probability amplitude for double occupancy is set to zero ${ }^{12}$. A formalism to deal with the Gutzwiller projection for the cuprates has recently been developed by one of $\mathrm{us}^{8}$, and was found to have the unusual property that there are no true quasiparticle excitations, that is, the quasiparticle residue at the Fermi level is zero. Despite this, here we show that 

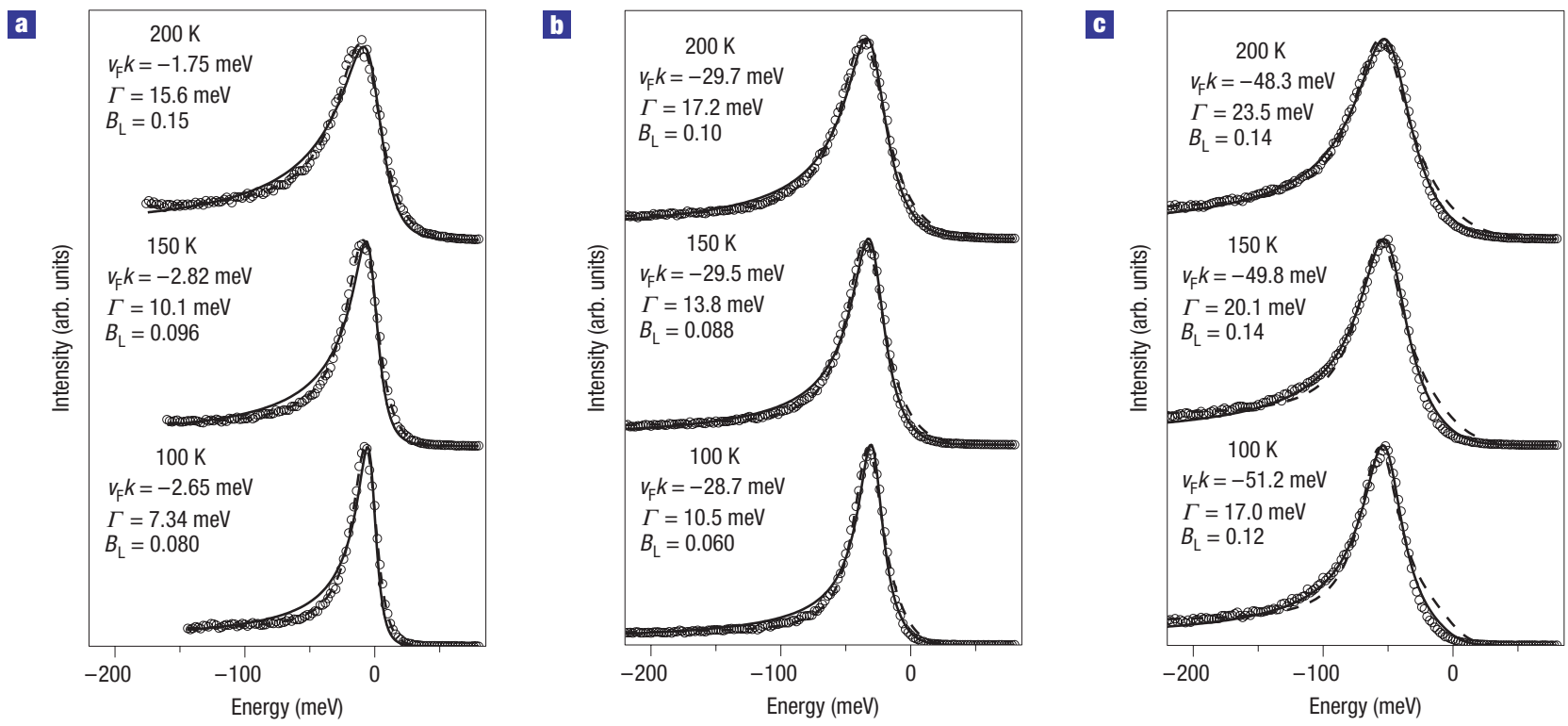

Figure 2 Laser-excited ARPES EDCs in the strange-metal phase above $T_{\mathrm{c}}$ of optimally doped $\mathrm{Bi}_{2} \mathrm{Sr}_{2} \mathrm{CaCu}_{2} \mathbf{O}_{8+\delta}$. a-c, $k$ near $k_{\mathrm{F}}(\mathrm{a})$ and at higher quasiparticle energies $(\mathbf{b}, \mathbf{c})$, as quoted. Data points are experimental and dashed curves are fitted lorentzians with background, $B_{\mathrm{L}} f(\omega / T)$. Solid curves are theoretical fits from the present paper, equation (1). Backgrounds are measured in units of the intensity relative to the peak of the EDC.

this quasiparticle-less theory produces excellent fits to the sharp laser-excited ARPES spectra, which look more quasiparticle-like than any previous ARPES spectra.

In ref. 8 , it was shown that the effect of the Gutzwiller projection is to multiply the free-particle Green's function in space-time by a factor $t^{-p}$, where $p$ is $(1 / 4)(1-x)^{2}$ and $x$ is the hole doping level. This value of the exponent is approximately confirmed by the exponent of the infrared conductivity dependence on frequency ${ }^{13}$, as shown in Fig. 1. Motion of a particle near the Fermi surface is essentially one-dimensional, so we may take the free-particle Green's function in space-time as $1 /\left(x-v_{\mathrm{F}} t\right)$. To get the imaginary part of $G$ (the density) in $k$ and frequency space, we must Fourier transform $G(x, t)$,

$$
G(k, \omega)=\iint \mathrm{d} x \mathrm{~d} t \mathrm{e}^{i(k x-\omega t)} t^{-p} /\left(x-v_{\mathrm{F}} t\right) .
$$

Doing the $x$ integration by a contour integration (the sign of $t$ determines which way to close the contour), this becomes,

$$
G(k, \omega)=\int \mathrm{d} t t^{-p} \mathrm{e}^{i\left(v_{\mathrm{F}} k-\omega\right) t} \propto\left(v_{\mathrm{F}} k-\omega\right)^{-1+p} .
$$

The imaginary part of this expression is the $T=0$ energy distribution curve (EDC). If $p=0$, this is just a delta function at the quasiparticle energy, $v_{\mathrm{F}} k$, but if $p$ is finite it has an imaginary part for all $\omega>v_{\mathrm{F}} k$. The quasiparticle becomes a cut singularity, not a pole, in the complex plane and does not have a finite residue at the singularity, that is, it has quasiparticle residue $Z=0$.

The absence of a finite $Z$ has a profound effect on the temperature behaviour. If there are ordinary quasiparticles, their energies are not affected by thermal fluctuations. Impurity scattering, for instance, only changes their spatial wavefunctionsand there is no tendency towards ' $\omega, T$ scaling', which we naively expect from the analogy between the Boltzmann distribution $\mathrm{e}^{-E / k_{\mathrm{B}} T}$ and the Schrodinger time dependence $\mathrm{e}^{i H t}$. In other words, in that case there are conservation laws, effected by Ward identities, which restrict the scattering, whereas in the current case there are only phase space restrictions, so that the effect of raising the energy is the same as raising the temperature. A heuristic approximation to the effect of finite $T$ is to insert a relaxation rate $\Gamma=A T$, where $A$ is a constant of order unity, so that we replace $t^{-p}$ by $t^{-p} \mathrm{e}^{-\Gamma t}$. Yuval has suggested that for finite $T, t \rightarrow \sinh (\pi T t) / \pi T$ (ref. 14), so that we might expect $A \sim \pi p$, but that is only an estimate. The excitations into which any decay takes place are fermionic, so that we must also multiply by the Fermi function of energy $h \omega / 2 \pi$.

The observed spectra clearly show a broadening that increases with increasing binding energy. A conventional Fermi liquid would have the scattering proportional to $\omega^{2}$, where $\omega$ is the difference from the Fermi energy, producing further decay for the curves with $k$ farther from $k_{\mathrm{F}}$. In a supplement for ref. 8, it is shown that there is an underlying, 'hidden' Fermi liquid of excitations, which can therefore be expected to be scattered at the conventional rate $\sim \omega^{2}$ (ref. 15). The final expression for the EDC then becomes,

$$
\begin{aligned}
\text { Intensity } & =\operatorname{Im}\{G\}=\operatorname{Im}\left\{\frac{f(\omega / T)}{\left[\left(v_{\mathrm{F}} k-\omega\right)+i \Gamma\right]^{1-p}}\right\} \\
& =f(\omega / T) \frac{\sin \left[(1-p)\left(\pi / 2-\tan ^{-1}\left[\left(\omega-v_{\mathrm{F}} k\right) / \Gamma\right]\right)\right]}{\left[\left(\omega-v_{\mathrm{F}} k\right)^{2}+\Gamma^{2}\right]^{(1-p) / 2}} .
\end{aligned}
$$

Here, we have expressed all frequencies and temperatures in the same energy units, and $f$ is the Fermi distribution,

$$
f(\omega / T)=\frac{1}{\mathrm{e}^{\hbar \omega / k_{\mathrm{B}} T}+1} .
$$

The line shape, equation (1), is the Doniach-Sunjic line shape ${ }^{16}$ multiplied by the Fermi function. Although it shares with the simple power law the fact that the singularity is not a pole and has $Z=0$, it was historically often mistaken for a simple lorentzian.

In principle, it is possible to calculate the temperature dependence of the Green's function, and its Fourier transform $G(k, \omega)$, in a less heuristic way, because Yuval has given a prescription for the Green's function in the X-ray problem at finite 


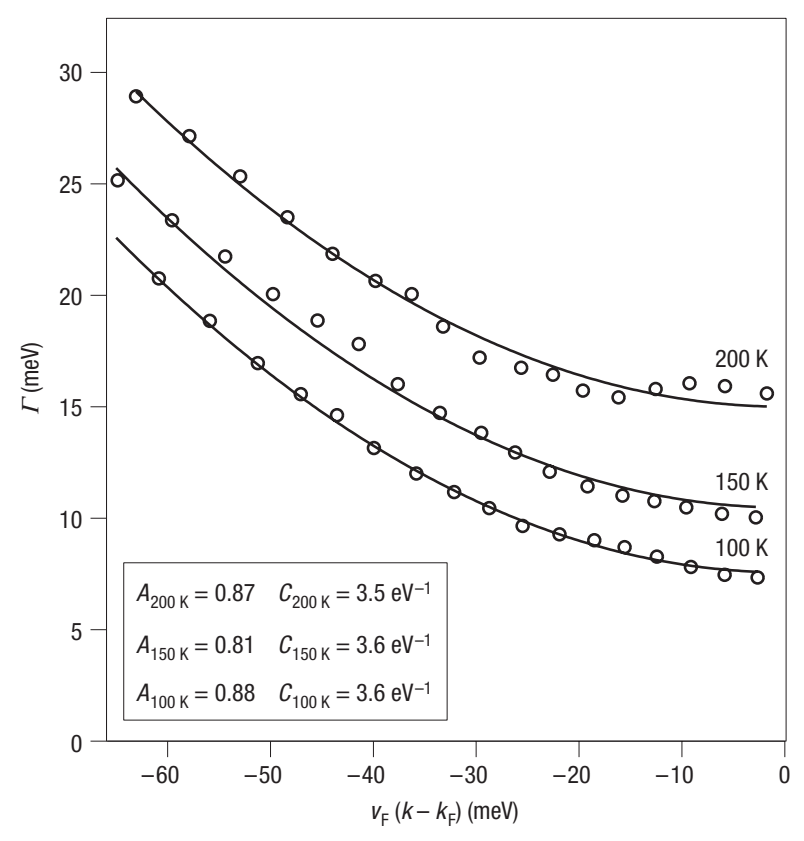

Figure 3 Relaxation rate, $\Gamma$, (data points) extracted by fitting the experimental EDCs. Fits were equation (1). Solid lines are best fits of $\Gamma=A k_{\mathrm{B}} T+C v_{\mathrm{F}}^{2}\left(k-k_{\mathrm{F}}\right)^{2}$. The given empirical values fit well to universal parameters $A=0.85$ and $C=3.6 \mathrm{eV}^{-1}$.

$T$ (ref. 14). However, the formalism would involve enormously complicated contour integration and we think the above heuristic form is adequate for the current purposes. In explicit form, equation (1) is quite complicated, and it should be: the central portion resembles a lorentzian, whereas the tail behaves like $\omega^{-1+p}$.

Figure 2 compares the theoretical predictions of equation (1) with the measured laser-excited ARPES EDCs at the three $k$ points and three temperatures from ref. 9 (many more $k$ points were fitted as seen in Fig. 3). Lorentzian fits to the data are also shown, similar to those given in ref. 9. The fits using equation (1) did not include any background term, whereas a small energyindependent background was added for the lorentzian fits, as this was necessary for them to obtain reasonable fidelity. A value $p=0.18$, corresponding to the optimal hole doping level $x=0.15$ was used. The sample was nearly optimally doped $\mathrm{Bi}_{2} \mathrm{Sr}_{2} \mathrm{CaCu}_{2} \mathrm{O}_{8+\delta}$ with a transition temperature of around $90 \mathrm{~K}$, and $k$ was along the nodal direction, so at the temperatures between 100 and $200 \mathrm{~K}$ there is little or no effect of the superconducting gap. The parameters for each curve are also given in Fig. 2.

The fits using equation (1) are superior to the pure lorentzian fits at capturing the asymmetry of the measured EDCs, especially for the spectra far from the Fermi energy, $E_{\mathrm{F}}$. In principle, these new fits have at most one truly free parameter-an approximation leaves one numerical coefficient free within narrow limits. The current fits are not perfect, nor should they be-the exponential cutoff assumed is probably too slow, and we might expect a steeper falloff on the low-energy side. We also note that no extra background term is needed to fit with the strange-metal line shape, whereas one is needed to fit the pure lorentzians (this background amplitude is reported in Fig. 2 as $B_{\mathrm{L}}$ ). We have found that the spectral functions of Fermi liquid and marginal Fermi liquid theories can also capture the asymmetry of the data better than pure lorentzians. However, these theories were not found to fit the data set across multiple temperatures with a universal set of parameters as the strange-metal theory does, and therefore a detailed discussion of them will be reserved for a future publication by J.D.K. et al.

Figure 3 shows the trends of the extracted relaxation rate, $\Gamma$, as a function of temperature and binding energy. A similar plot for the lorentzian fits can be seen in Fig. 3b of ref. 9. The current fits show a slightly smaller $\Gamma$ and a smoother behaviour, which we parameterize as $\Gamma=A k_{\mathrm{B}} T+C v_{\mathrm{F}}^{2}\left(k-k_{\mathrm{F}}\right)^{2}$. As seen in Fig. 3 , the extracted $A$ and $C$ values are nearly constant over all the individual EDC fits, implying a near-universal set of fits.

The spectral line shapes predicted by the strange-metal theory of cuprate superconductors are found to fit the laser-excited ARPES EDCs accurately and with a minimal number of free parameters. Compared with simple lorentzian fits, the asymmetry in the EDCs is well characterized and no background term is required. It should now be possible, with considerably more computational effort, to analyse the curves in the superconducting state and understand the subtle intensity transfers that occur there.

Received 18 July 2007; accepted 6 December 2007; published 13 January 2008

\section{References}

1. Anderson, P. W. The Theory of Superconductivity in the High $-T_{\mathrm{c}}$ Cuprates (Princeton Univ. Press, Princeton, 1997).

2. Varma, C. M., Littlewood, P. B., Schmitt-Rink, S., Abrahams, E. \& Ruckenstein, A. Phenomenology of the normal state of $\mathrm{Cu}-\mathrm{O}$ high-temperature superconductors. Phys. Rev. Lett. 63, 1996-1999 (1989). ibid 64, 497(E) (1990).

3. Orenstein, J. \& Millis, A. J. Advances in the physics of high-temperature superconductivity. Science 288, 468-474 (2000)

4. Batlogg, B. A critical review of selected experiments in high- $T_{\mathrm{c}}$ superconductivity. Physica $B \mathbf{1 6 9}$ 7-16 (1991).

5. Coffey, D., Meltzer, D. E., Pines, D., Bedell, K. S. \& Schrieffer, J. R. (eds) High Temperature Superconductivity: Proceedings: The Los Alamos Symposium, 1989 37-82 (Addison-Wesley, Reading, 1990)

6. Shen, Z. X. \& Dessau, D. S. Electronic structure and photoemission studies of late transition-metal oxides-Mott insulators and high-temperature superconductors. Phys. Rep. 253, 1-162 (1995).

7. Damascelli, A., Hussain, Z. \& Shen, Z. X. Angle-resolved photoemission studies of the cuprate superconductors. Rev. Mod. Phys. 75, 473-541 (2003).

8. Anderson, P. W. The 'strange metal' is a projected Fermi liquid with edge singularities. Nature Phys. 2, 626-630 (2006)

9. Koralek, J. D. et al. Laser based angle-resolved photoemission, the sudden approximation, and quasiparticle-like spectral peaks in $\mathrm{Bi}_{2} \mathrm{Sr}_{2} \mathrm{CaCu}_{2} \mathrm{O}_{8+\delta}$. Phys. Rev. Lett. 96, 017005 (2006).

10. Koralek, J. D. et al. Experimental setup for low-energy laser-based angle resolved photoemission spectroscopy. Rev. Sci. Instrum. 78, 053905 (2007).

11. Hüfner, S. Photoelectron Spectroscopy: Principles and Application (Springer, Berlin, 1995)

12. Gutzwiller, M. C. Effect of correlation on the ferromagnetism of transition metals. Phys. Rev. Lett. 10 159-162 (1963).

13. Hwang, J., Timusk, T. \& Gu, G. D. Doping dependent optical properties of $\mathrm{Bi}_{2} \mathrm{Sr}_{2} \mathrm{CaCu}_{2} \mathrm{O}_{8+8}$. J. Phys. Condens. Matter 19, 125208 (2007).

14. Yuval, G. \& Anderson, P. W. Exact results for the Kondo problem: One-body theory and extension to finite temperature. Phys. Rev. B 1, 1522-1528 (1970).

15. Anderson, P. W. Hidden Fermi liquid: The secret of high $T_{\mathrm{c}}$ cuprates. Preprint at $<$ http://www.arxiv.org/abs/0709.0656> (2007).

16. Doniach, S. \& Sunjic, M. Many-electron singularity in X-ray photoemission and X-ray line spectra from metals. J. Phys. C: Solid State Phys. 3, 285-291 (1970).

\section{Acknowledgements}

P.A.C. acknowledges support from a National Sciences and Engineering Research Council of Canada Postgraduate Scholarship. Support for J.D.K., N.C.P. and D.S.D. came from DOE Grant No. DE-FG0203ER46066, with other funding from NSF Grant No. DMR 0706657, and by the NSF ERC for Extreme Ultraviolet Science and Technology under NSF Award No. 0310717. J.D.K., N.C.P. and D.S.D. thank J. F. Douglas, Z. Sun, A. Fedorov, J. Griffith, S. Cundiff, M. Murnane and H. Kapteyn for help with the experiments and Y. Aiura, H. Eisaki and K. Oka for growing the Bi2212 samples. Correspondence and requests for materials should be addressed to P.W.A.

Reprints and permission information is available online at http://npg.nature.com/reprintsandpermissions/ 